\title{
GW Librae: A Unique laboratory for pulsations in an accreting white dwarf
}

\section{Odette Toloza Castillo*}

University of Warwick, Coventry, UK

E-mail: O.F.C.Toloza@warwick.ac.uk

\section{Boris T. Gänsicke}

University of Warwick, Coventry, UK

E-mail: Boris.Gaensicke@warwick.ac.uk

\section{JJ Hermes}

University of North Carolina, Chapel Hill, USA

E-mail: jjhermes@unc.edu

\section{Dean M. Townsley}

University of Alabama, Tuscaloosa, USA

E-mail: Dean.M. Townsleydua.edu

\section{Matthias R. Schreiber}

Universidad de Valparaíso, Valparaiso, Chile

E-mail: matthias@dfa.uv.cl

\begin{abstract}
Non-radial pulsations have been identified in a number of accreting white dwarfs in cataclysmic variables. These stars offer insight into the excitation of pulsation modes in atmospheres with mixed compositions of hydrogen, helium, and metals, and the response of these modes to changes in the white dwarf temperature. Among all pulsating cataclysmic variable white dwarfs, GW Librae stands out by having a well-established observational record of three independent pulsation modes that disappeared when the white dwarf temperature rose dramatically following its 2007 outburst. Our analysis of HST/COS ultraviolet spectroscopy taken in 2002, 2010 and 2011 showed that pulsations produce variations in the white dwarf effective temperature, as predicted by theory. Additionally, in May 2013 we obtained new HST ultraviolet observations that displayed unexpected behaviour: besides variability at $\simeq 275$ s, which is close to the post-outburst pulsations detected with HST in 2010 and 2011, also the white dwarf exhibits high-amplitude variability on $\mathrm{a} \simeq 4.4 \mathrm{~h}$ time-scale. We demonstrate that this variability is related to an increase of the photospheric temperature, argue against a short-lived accretion episode as the explanation, and discuss this event in the context of non-radial pulsations on a rapidly rotating star.
\end{abstract}

The Golden Age of Cataclysmic Variables and Related Objects - III, Golden2015

7-12 September 2015

Palermo, Italy

\footnotetext{
*Speaker.
} 


\section{Introduction}

A handful of He rich white dwarfs (WDs) in dwarf novae can drive non-radial pulsations at temperatures $>15000 \mathrm{~K}$ [24], carrying information about their interior. These WDs are heated during outbursts [14], and subsequently cool back, evolving through the instability strip in only a few years. GW Librae (GW Lib) is the prototypical DN that exhibits non-radial pulsations in quiescence near 650, 370, and $230 \mathrm{~s}$ [28]. Follow-up HST observations confirmed the pulsation origin: the power spectrum showed similar signals, but with amplitudes $\sim 10$ times higher than in the optical [19]. Such a wavelength-dependent amplitude is due the UV wavelengths covering the peak of the exponential Wien tail, where the flux is more sensitive to changes in the temperature [15]. GW Lib underwent a outburst in 2007 and the stable pulsation signals were immediately swamped by the light from the accretion disk. To date, several prominent signals have recurrently shown up, a short period signal near 290-300 s [6, 20], and relatively long periods $(\sim 19 \mathrm{~min}, \sim$ $2 \mathrm{~h}$, and $\sim 4 \mathrm{~h} ;[16,5,25])$. The shortest period signal was first detected in 2008 in the optical [6] and then with $H S T$ UV observations in 2010 and 2011 [20]. The longest period signal ( $\simeq 4 \mathrm{~h}$ ) was first seen at optical and UV wavelengths in 2008 increasing in amplitude in the following years $[16,5,25]$. Their studies agree that it is a recurrent signal that wanders in phase and amplitude.

Here we present new observations of GW Lib with the Cosmic Origin Spectrograph (COS).

\section{OBSERVATIONS}

\subsection{Ultraviolet spectroscopy}

We obtained UV time-tagged spectroscopy of GW Lib using COS/G140L (red line in Fig. 1). Additionally, we retrieved from the MAST archive all STIS and COS UV time-tag data taken with the G140L gratings: four STIS orbits taken in 2002 (for more details see [19]), and a total of three COS post-outburst orbits (see [20]). The average spectra are shown in left panel of Fig. 1.

The average spectrum of each epoch is dominated by the WD. However, it is clear that there is some flux contribution of an additional flat and featureless component since the core of $\operatorname{Ly} \alpha$ does not reach zero. Such component has been identified in many other DNe [10, 13, 18, 8], in all cases contributing $\lesssim 20 \%$ in flux, but the exact origin is not well understood.

\subsection{Variability}

We built UV light curves binning the time-tag data in $10 \mathrm{~s}$ (right panel in Fig. 1). While in 2002, 2010, and 2011 the system showed periodic variations due to non-radial pulsations [19, 20], the light curve of 2013 data is dominated by a large-amplitude variability (Fig. 1).

It is not known if the large-amplitude variability is cyclical or not, nevertheless we fitted the entire light curve with a sinusoidal function plus harmonics, using PERIOD04 [12]. The best fit is $4.39 \pm 0.09 \mathrm{~h}$. This period is close to the $\simeq 4 \mathrm{~h}$ period previously detected in the UV and optical $[16,5,25]$. In addition to the large-amplitude variability, the light curve shows periodic fluctuations with the highest amplitude in the second orbit. We performed Fourier Transform of the light curve for each HST orbit after subtracting the larger amplitude variability by fitting a second order polynomial independently to each orbit. In each orbit the strongest peak is found in the range $270-290$ s, with a statistically significant detection in the second and third orbits (Fig. 2). The 

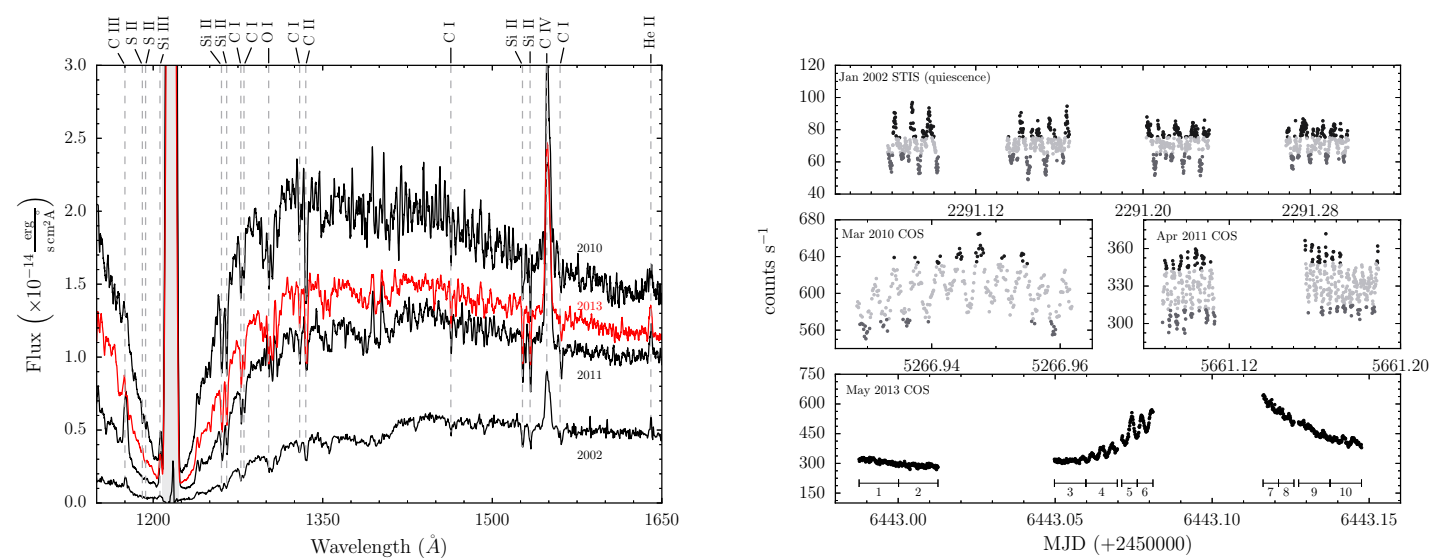

Figure 1: Left: Average COS and STIS spectra of GW Lib (no flux offsets were applied). Black lines from top to bottom show the observations from 2010, 2011 (both COS), and 2002 (STIS). The red line corresponds to the new 2013 COS observations, which unexpectedly show a higher flux level than the 2011 observations. The most prominent lines are labelled and the $\operatorname{Ly} \alpha$ airglow emission is shaded in light grey. Right: Light curves of GW Lib . From the observations obtained in 2002, 2010, and 2011, we extracted two spectra, one peak spectrum constructed from the intervals with the highest count rate (black dots), and one trough spectrum from those with the lowest count rate (dark grey dots). For the 2013 observation a set of ten spectra was created, as labelled underneath the light curve. We performed spectral fits to them (see Sect. 3).

periods are close to the 293 s period detected in 2010 and 2011, so we suggest they correspond to WD pulsations.

\section{Spectral fitting}

\subsection{2, 2010, and 2011 observations}

The excitation of $g$-modes, which occurs in the $\mathrm{H}(\mathrm{He})$ ionisation zones, is produced by an increase of the opacity generating a convecting zone [4]. The dominant effect of the pulsations is the appearance of hot and cool patterns on the WD surface [15]. The three well-defined period pulsations identified during quiescence, as well as the $293 \mathrm{~s}$ period found post-outburst data, are generally believed to be due to non-radial pulsations. Therefore we investigated the difference in temperature produced by these pulsations. To that aim, we performed spectral fits to two spectra, for each of the 2002, 2010, and 2011 observations. One spectrum was constructed using the photons corresponding to the sections of the light curve with the highest count rate (black dots in Fig. 1) and a second spectrum from the sections with the lowest count rate (dark grey dots in Fig. 1), hereafter referred to as peak and trough spectra. These spectra were obtained using a series of PyRAF routines from the STSDAS package and modules of the STSCI_PYTHON2.15 library.

We used a grid of WD models generated with TLUSTY195 and SYNSPEC45 [11]. We fixed $\log g=8.35$, corresponding to the WD mass estimate for GW Lib $\left(M_{\mathrm{WD}}=0.84 \pm 0.02 \mathrm{M}_{\odot}\right.$; [27]). We used a metallicity of 0.1 solar, which reproduces well the metal absorption lines.

The core of the photospheric $\operatorname{Ly} \alpha$ absorption line shows evidence of an additional flat and featureless continuum component. Therefore we find the effective temperature, the scaling factor, 


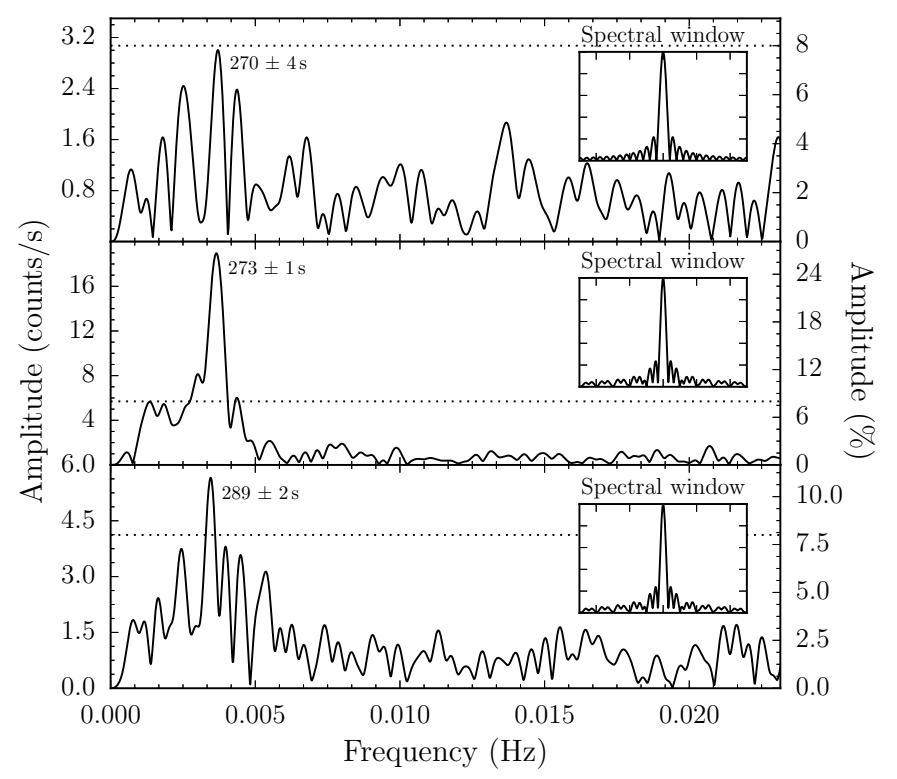

Figure 2: Power spectra for each orbit of the 2013 HST observations. The significance level (dotted line) was defined as four times the mean of the amplitude calculated from 0 up to the Nyquist frequency.

Table 1: Best-fit effective temperatures, with a fixed $\log g=8.35$.

\begin{tabular}{ccccccc}
\hline \hline State & $T_{\text {eff, } 2002}(\mathrm{~K})$ & $T_{\text {eff, } 2010}(\mathrm{~K})$ & $T_{\text {eff, } 2011}(\mathrm{~K})$ & $R_{\mathrm{WD}, 2002}\left(\mathrm{R}_{\odot}\right)$ & $R_{\mathrm{WD}, 2010}\left(\mathrm{R}_{\odot}\right)$ & $R_{\mathrm{WD}, 2011}\left(\mathrm{R}_{\odot}\right)$ \\
\hline Trough & $14698_{-25}^{+25}$ & $18374_{-74}^{+74}$ & $16085_{-68}^{+70}$ & $0.007_{-0.001}^{+0.002}$ & $0.008_{-0.002}^{+0.002}$ & $0.009_{-0.002}^{+0.003}$ \\
Peak & $15187_{-20}^{+22}$ & $18834_{-69}^{+66}$ & $16565_{-52}^{+52}$ & $0.007_{-0.001}^{+0.002}$ & $0.008_{-0.002}^{+0.002}$ & $0.008_{-0.002}^{+0.003}$ \\
\hline
\end{tabular}

and an additional continuum component flat in $F_{\lambda}$ which best fit the peak and trough spectra. We adopted a flat component as it reduces the total number of free parameters.

We used the EMCEE code ${ }^{1}$. We constrained $T_{\text {eff }}$ over the range $10000-20000 \mathrm{~K}[19,20]$, as well as $k$ and $S$ to be positive. Finally we defined the log-likelihood function to be $-\chi^{2} / 2$. To estimate an initial guess for the parameters we used the Levenberg-Marquardt minimization method. We masked the Ly $\alpha$ airglow line and the $\mathrm{C}$ IV emission lines during the fit.

The best fit effective temperatures for the peak and trough spectra of 2002, 2010, and 2011 are listed in Table 1 where we calculated the internal uncertainty as the 15.87 and 84.1 percentiles of the posterior. The results show that the pulsations led to a difference of nearly $500 \mathrm{~K}$.

We derived the WD radius using the scaling factor $(S)$ and the distance of $104_{-20}^{+30} \mathrm{pc}$ [21]. The resulting radii are listed in Table 1, where the large uncertainties are primarily systematic, resulting from the error on the distance measurement. Within the uncertainties, the derived radii agree with the expected radius of $\mathrm{a} \simeq 0.84 \mathrm{M}_{\odot} \mathrm{WD}$ located at the distance of $\mathrm{GW} \mathrm{Lib}^{2}$

\footnotetext{
${ }^{1}$ http://dan.iel.fm/emcee/current/

${ }^{2}$ The radius derived from DA cooling models [2] http://www.astro.umontreal.ca/ bergeron/CoolingModels
} 


\subsection{3 observation}

While we expect that WD pulsations cause the short-period oscillations seen in the light curve, the nature of the $\simeq 4.4 \mathrm{~h}$ long flux variability is unclear. Here, we explore its origin in the WD photosphere. For that purpose, we process the 2013 COS data into a set of ten spectra, as labelled underneath the light curve in the bottom panel of Fig. 1. In each of the spectra the UV flux is well-described by the WD model plus a small continuum flux component. We then fitted this set of spectra following the same procedure as in Sect. 3.1.

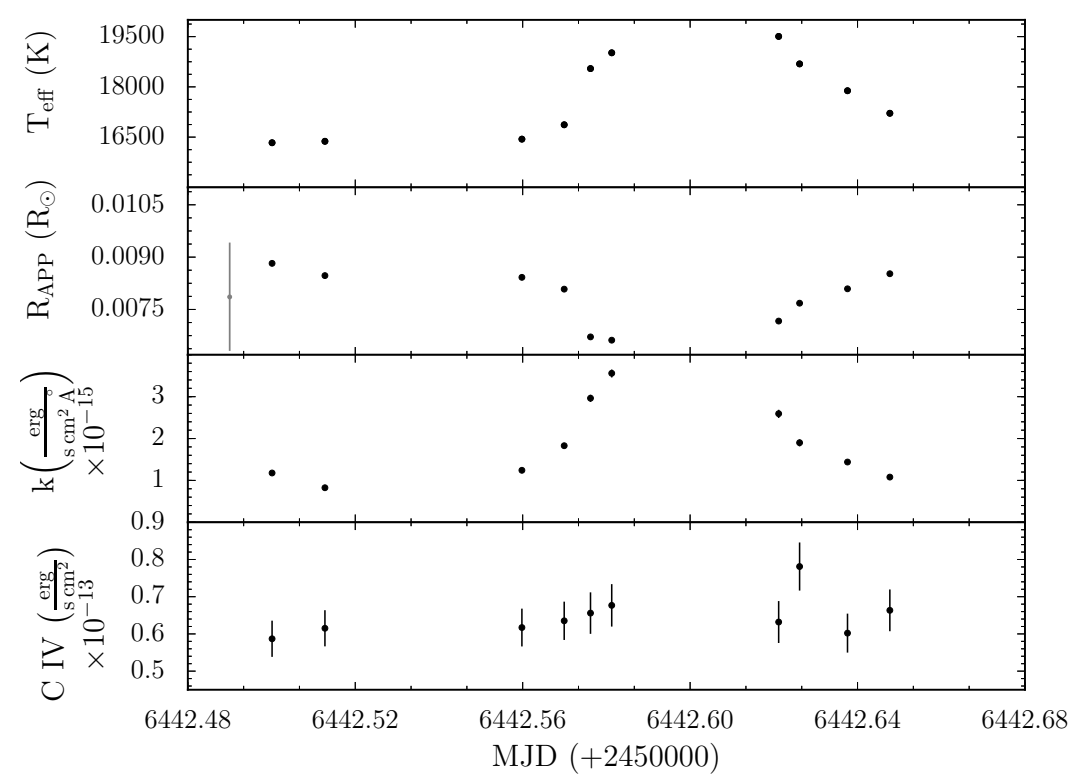

Figure 3: Best-fit parameters for the set of ten spectra processed from the May 2013 observations. The intrinsic uncertainties have been estimated based on the 13.59 and 84.1 percentiles of the posterior, but they are too small to be seen in this scale. The distance measurement used to determine the apparent WD radius introduces a large systematic uncertainty (gray dot). This systematic uncertainty affects, however, the entire sequence of apparent radii in the same way. The fourth panel shows the flux of C IV emission line at $1550 \AA$. See Section 4 for details.

The best-fit parameters for the ten spectra are illustrated in Fig. 3. The top panel shows that the effective temperature of the WD varies from $16332_{-25}^{+22} \mathrm{~K}$ to $19506_{-59}^{+51} \mathrm{~K}$. The second panel illustrates the apparent WD radius obtained from the scaling factor. We adopt the designation of apparent radius as a change in the actual WD radius is unphysical. However, if the temperature of the WD rises over a localised region on its surface, this region will dominate the UV flux, resulting in a decrease of the apparent radius, which is exactly what we find. We note, however that, the apparent WD radius derived from spectrum \#2, i.e. near the minimum flux of the 2013 observations, is $0.008_{-0.002}^{+0.002} \mathrm{R}_{\odot}$, consistent with the radius estimates from the 2002,2010 , and 2011 observations (Table 1). The large uncertainty is again due to the systematic error associated with the distance [21]. The third panel shows the additional constant flux model, which follows a similar behaviour of the effective temperature (top panel). Additionally we show the flux of the C IV emission line in the fourth panel (discussed below). 


\section{DISCUSSION}

We demonstrated that the evolution of both the total UV flux, as well as the morphology of the Ly $\alpha$ profile obtained in 2013, is well-described by an increase and subsequent decrease of the temperature of significant fraction of the visible WD surface. Here we discuss possible scenarios that could cause such change in the WD temperature.

\subsection{An accretion spot on a magnetic WD.}

Accretion causes heating of the WD, and non-uniform accretion of matter will result in an inhomogeneous temperature distribution over the WD surface. Accretion-heated spots that exceed the temperature of the underlying WD by several $1000 \mathrm{~K}$ are frequently observed in polars, i.e. strongly magnetic CVs, and cause a modulation of the ultraviolet flux and the width of the Ly $\alpha$ profile on the spin period of the WD $[7,9,17,1]$. Assuming the presence of a weak magnetic field (B) we can calculate the minimum magnitude required by the WD to decouple the electron spin and detect a splitting of sharp metal lines given the spectral resolution of COS. We conclude that, because we do not detect Zeeman splitting in Si II at $1265 \AA$, the magnetic field of the WD in GW Lib is $B \leq 0.9 \mathrm{MG}$. However, in polars, the rotation of the WD is locked to the orbital period, in the range $\simeq 1.5-8 \mathrm{hr}$, whereas the spin period of GW Lib is $\simeq 100-200 \mathrm{~s}[27,20]$. Hence, accretion onto a magnetic WD would result in coherent variability on time scales of $\simeq 100-200 \mathrm{~s}$, and we therefore rule out this scenario to explain the $\simeq 4 \mathrm{~h}$ variability.

\subsection{A brief increase in the accretion rate.}

Quasi-periodic brief accretion events could heat a fraction of the WD. However, the accretion needs to be extremely symmetric with respect to the spin axis of the WD, as we do not observe any variability of the UV flux at the WD spin period.

We can estimate the excess of energy released during a hypothetical accretion episode from the excess luminosity. We derived the luminosity by integrating WD models that follow the temporal evolution of the effective temperature shown in the top panel of Fig. 3, scaled to the observed flux and subtracting the flat component. The energy excess corresponds to the area above of the luminosity of the underlying WD $\left(T_{\mathrm{eff}}=16439 \mathrm{~K}\right.$, blue line). Adopting $M_{\mathrm{WD}}=0.84 \mathrm{M}_{\odot}$ [27] and $R_{\mathrm{WD}}=0.008 \mathrm{R}_{\odot}$ (See section 3.2) we can derive a total mass accreted during a short episode (Fig. 4). The discontinuity of the HST data does not allow to accurately define the shape of such area. The simplest interpolation is connecting the points with a straight line, giving a total accreted mass of $\simeq 1.5 \times 10^{-16} \mathrm{M}_{\odot}$ in $\simeq 2.1 \mathrm{hr}$, which is a strict lower limit. Alternatively, we fit the luminosity adopting the same model we used to fit the light curve (red solid line). This approach gives an accreted mass of $2.0 \times 10^{-16} \mathrm{M}_{\odot}$ in $\simeq 2.6 \mathrm{~h}$. Between the two approaches, we found a lower accretion rate $\left(6.3-6.6 \times 10^{-13} \mathrm{M}_{\odot} / \mathrm{yr}\right)$, than the expected for DNe in quiescent state [22].

In principle, small changes in the accretion rate could be caused by efficient irradiation of an ionised inner disc by the WD, while the outer disc is cooler and neutral. Instabilities in the transition region could cause quasi-periodic fluctuations of the accretion rate. Another possibility is that a twisting and reconnecting magnetic field between star-disc can cause quasi periodic changes of the accretion rate in the inner disc [23]. One may speculate that such cycles can generate quasi-periodic episodes of enhanced accretion. 
If the $\simeq 4 \mathrm{~h}$ variability is related to quasi-periodic brief accretion events, one would expect similar activity in additional CVs. [26] and [25] note that only a handful of other systems show quasi-periodic variability at periods longer than their orbital periods. Although quasi-periodic accretion events are in principle a possible explanation, we consider them unlikely because firstly, we would expect an increase in the accretion rate onto the WD to be accompanied with photometric flickering, and secondly, enhanced accretion activity should be linked to a variation of the C IV emission line flux. Both are not observed (Fig. 3).

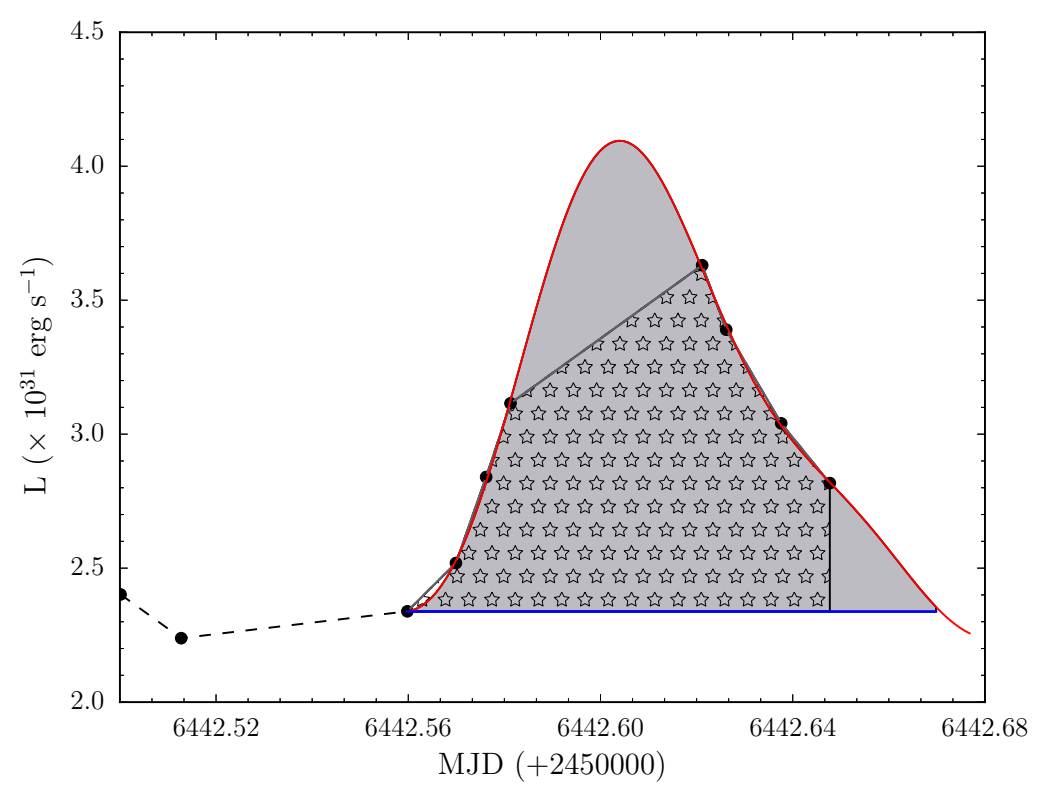

Figure 4: Luminosity as a function of time calculated from integrating the flux of WD models with the effective temperatures shown in Table 1. The starred area represents an excess of energy of $6.0 \times 10^{34} \mathrm{ergs}$ while the grey area corresponds to $7.9 \times 10^{34}$ ergs.

\subsection{Retrograde wave in a rapid rotating WD}

In the case of rapid rotation, $\Omega_{\text {spin }}>\Omega_{\text {mode }}$, the "splitting" of $g$-modes by rotation is no longer a small perturbation. Also, modes on rotating stars are sensitive to the rotation axis and the direction of rotation. As a result, for $l=1$ modes, the modes that corresponds to the $m=-1$ spherical harmonics propagate in the same direction as the spin, while the $m=+1$ mode corresponds to retrograde modes. For the retrograde modes, the frequency measured by a fixed observer is $\Omega_{\mathrm{obs}}=$ $\left|\Omega_{\text {mode }}-\Omega_{\text {spin }}\right|$. The $g$-mode spectrum will be a sequence of modes starting at around a few hundred seconds and extending to longer periods as the radial order of the mode increases [3]. As a result, for a star like GW Lib, which is rotating with a period of around $100-200$ seconds [27, 20], there can be a low-radial-order $g$-mode, $n \sim 5$, for which $\Omega_{\text {mode }} \approx \Omega_{\text {spin }}$ so that $\Omega_{\text {obs }}$ is closer to zero, thus giving a significantly longer period (Fig. 5).

The rotationally modified $g$-modes do not extend evenly over the entire surface of the star. Their amplitudes are larger near the equatorial regions [3], depending of the ratio between $\Omega_{\text {mode }}$ and $\Omega_{\text {spin. }}$. Lower frequency (higher radial order) modes are constrained more tightly near the 


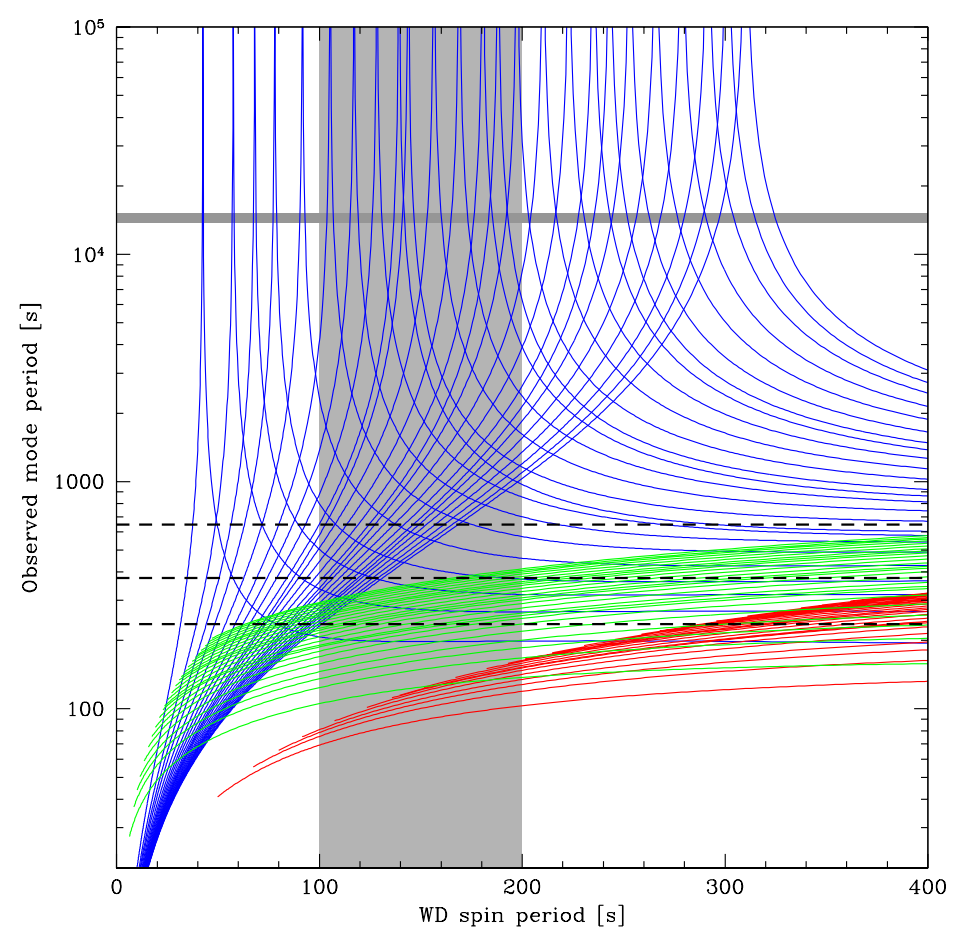

Figure 5: Observed mode period produced by the splitting of the $g$-modes due to fast rotating WD, where $\mathrm{m}=1$ (retrograde, blue), $\mathrm{m}=-1$ (prograde, red), and $\mathrm{m}=0$ (green). This model was computed using a total mass, $M=0.9 \mathrm{M}_{\odot}$, a H-rich accreted layer mass of half of the nova ignition mass, and $T_{\text {eff }}=15500 \mathrm{~K}$ [19]. The dashed lines show the 646, 376, and 237 s periods seen during quiescence. The light shaded represents the WD spin period $[27,20]$ and the dark gray band corresponds to the observed $\simeq 4.4 \mathrm{~h}$-period variability.

equator. The moderate radial order necessary to have $\Omega_{\text {spin }} \approx \Omega_{\text {mode }}$ could therefore also lead to the decrease in the effective area as the mode contributes more to the flux. Some of this contribution may be blocked by the edge-on accretion disk, but if the mode extends to high enough latitude to be visible it would lead to a reduction in area like that observed.

The mode responsible for the $\simeq 4 \mathrm{~h}$ variability is not continuously detected, because it was not excited or if its period shifts by a small amount it may no longer be similar to the spin period.

\section{Conclusion}

We have shown that the non-radial pulsations in GW Lib lead to variations of a few hundred Kelvin over the visible WD surface. In the new 2013 COS observations, we identified again the presence of pulsations with period $\sim 275-290 \mathrm{~s}$, and a large amplitude variability with a period of $\simeq 4.4 \mathrm{~h}$. We suggest to be the same $\simeq 4 \mathrm{~h}$ signal previously detected in the UV and optical by $[16,5$, 25]. We demonstrated that the $\simeq 4.4 \mathrm{~h}$ variability is produced by a simultaneous increase of the WD temperature and a decrease of its apparent radius. Subsequently, the WD cools while the apparent radius is relaxing back to its original configuration. We postulate that this large temperature change occurs on a fraction of the WD surface. A wave travelling opposite to the direction of the WD rotation, with a period similar to the spin period is most plausible. 


\section{References}

[1] Araujo-Betancor et al., Far-Ultraviolet Spectroscopy of Magnetic Cataclysmic Variables, 2005, ApJ, 622, 589

[2] Bergeron, P. et al., A Comprehensive Spectroscopic Analysis of DB White Dwarfs, 2011, ApJ, 737, 28

[3] Bildsten, L. et al., Ocean g-Modes on Rotating Neutron Stars, 1996, ApJ, 460, 827

[4] Brickhill A. J. The pulsations of ZZ Ceti stars, 1983, MNRAS, 204, 537

[5] Bullock E. et al., GALEX and Optical Observations of GW Librae during the Long Decline from Superoutburst, 2011, AJ, 141, 84

[6] Copperwheat C. M. et al., ULTRACAM observations of two accreting white dwarf pulsators, 2009, MNRAS, 393,157

[7] Gänsicke B. T. et al., HST/GHRS observations of AM Herculis 1998, 338, 933

[8] Gänsicke B. T. et al., Hubble Space Telescope STIS Observations of the Accreting White Dwarfs in BW Sculptoris, BC Ursae Majoris, and SW Ursae Majoris, 2005, ApJ, 629, 451

[9] Gänsicke B. T. et al. FUSE and HST STIS Far-Ultraviolet Observations of AM Herculis in an Extended Low State, 2006, ApJ, 639, 1039

[10] Godon P. et al., Far-Ultraviolet Observations of the Dwarf Nova VW Hydri in Quiescence, 2004, ApJ, 612, 429

[11] Hubeny I. \& Lanz T., Non-LTE line-blanketed model atmospheres of hot stars. 1: Hybrid complete linearization/accelerated lambda iteration method, 1995, ApJ, 439, 875

[12] Lenz P. \& Breger M., Communications in Asteroseismology, 2005, CoAst, 146, 53

[13] Long K. S. et al., The Effect of a Superoutburst on the White Dwarf and Disk of VW Hydri as Observed with FUSE, 2009, ApJ, 697, 1512

[14] Piro A. L. et al., White Dwarf Heating and Subsequent Cooling in Dwarf Nova Outbursts, 2005, ApJ, 628, 401

[15] Robinson E. L., et al., The pulsation index, effective temperature, and thickness of the hydrogen layer in the pulsating DA white dwarf G117-B15A, 1995, ApJ, 438, 908

[16] Schwieterman E. W., et al., Time-Series Photometry of GW Librae One Year After Outburst, 2010, Journal of the Southeastern Association for Research in Astronomy, 3, 6

[17] Schwope A. D. et al., A multiwavelength timing analysis of the eclipsing polar DP Leo, 2002, 392, 541

[18] Sion E. M. et al., Hubble Space Telescope STIS Spectroscopy of the White Dwarfs in the Ultrashort-Period Dwarf Novae VY Aquarii and WX Ceti 2003, ApJ, 583, 907

[19] Szkody P. et al., Hubble Space Telescope Spectra of GW Librae: A Hot Pulsating White Dwarf in a Cataclysmic Variable, 2002, ApJ, 575, L79

[20] Szkody P., et al., HST and Optical Data Reveal White Dwarf Cooling, Spin, and Periodicities in GW Librae 3-4 Years after Outburst, 2012, ApJ, 753, 158

[21] Thorstensen J. R., Parallaxes and Distance Estimates for 14 Cataclysmic Variable Stars, 2003, AJ, 126, 3017

[22] Townsley D. M. \& Gänsicke B. T., Cataclysmic Variable Primary Effective Temperatures: Constraints on Binary Angular Momentum Loss, 2009, ApJ, 693, 1007

[23] Uzdensky D. A., Partial Field Opening and Current Sheet Formation in the Disk Magnetosphere, 2002, ApJ, 572,432

[24] Van Grootel V., Fontaine G., Brassard P., Dupret M.-A., 2015, A\&A, 575, A125

[25] Vican L., et al., 2011, PASP, 123, 1156

[26] Woudt P. A. \& Warner B., A 2.09-h Photometric Periodicity in GW Librae, 2002, Ap\&SS, 282, 433

[27] van Spaandonk L. et al., The Mass of the White Dwarf in GW Libra 2010, ApJ, 715, L109

[28] van Zyl L. et al., GW Librae: An Accreting Variable White Dwarf, 2000, Baltic Astronomy, 9, 231 